\title{
Real-Time Experimental Connection of an EnergyPlus Building Model With a Real Water-to- Water Heat Pump Through Functional Mock-Up Interface
}

\author{
Paolo Taddeo $^{1}$, Ivan Bellanco ${ }^{1}$, Elena Fuentes ${ }^{1}$, Joana Ortiz ${ }^{1,2}$, Jaume Salom ${ }^{1}$ \\ ${ }^{1}$ Catalonia Institute for Energy Research (IREC), Jardins de les Dones de Negre 1, 08930 Sant \\ Adrià de Besòs (Barcelona), Spain \\ ${ }^{2}$ Universitat Politècnica de Catalunya, Barcelona, Spain
}

\begin{abstract}
Energy modelling is a powerful tool to predict building physical performance. However, current simulation tools are not able to mimic perfectly the operation of real HVAC systems. Testing real HVAC equipment under the loads of a virtual model makes it possible to analyse their dynamic behaviour under quasi-realistic conditions. New methods are needed to exchange information between building modelling tools and laboratories where real systems are tested. Within this context, the present study focuses on the implementation of a data communication chain to perform real-time building simulation in EnergyPlus with hardware-in-loop (HIL) integration in a semi-virtual testing laboratory (SEILAB). A feature of SEILAB is that it allows operating real equipment under the energy demand/production of a dynamic virtual building model. A residential building model created with EnergyPlus has been coupled with a real water-to-water heat pump, an on-site weather station and a photovoltaic system. This communication is based on the use of Functional Mock-up Interface for Co-Simulation (FMI). The EnergyPlus model has been wrapped into a Functional Mock-up Unit (FMU) that communicates with the laboratory by means of LabVIEW. The latter provides a graphical interface of SEILAB to manage the real equipment. Coupling between EnergyPlus and LabVIEW requires Python's scripts as a bridge for communication.
\end{abstract}

\section{Introduction}

Energy consumption due to heating and cooling needs is a large portion in residential and commercial buildings. Therefore, the reduction of energy use would be mostly valuable for improving the performance of such buildings. Because of the increasing implementation of heat pumps in installations around the world, it is necessary to investigate on strategies for their better integration and regulation. Laboratory testing on the integration of heat pumps using different strategies is required as there is a lack of experimental studies that investigate the effect of control operation on these systems (Waddicor, 2016). Laboratory experimentation using a hardware-in-loop (HIL) configuration improves tests repeatability, allowing operating the heat pump as if it was installed in a real facility under different conditions. HIL simulations present several advantages compared to field tests as they require less effort on the implementation and they allow more accuracy in the measurements.
Therefore, conclusions can be drawn with less time and effort under several different scenarios.

The connection between the heat pump and the model is done using communication protocols that allow data transfer between the real equipment and a simulation model that reproduces the thermal loads of a virtual building. Few studies address the design and definition of communication methods between the different heating systems. El-Baz et al. (2018), after a comprehensive review about HIL implementation, present a HIL system of a heat pump testbed, controlled with a LabVIEW program and its data communication protocol with a building model built in SimulationX, a Modelica based program. It shows that the HIL system is able to maintain realistic dynamics. However, there are no studies that deal with a HIL heating system and the open software EnergyPlus, which is widely used in the building simulation community. Jones et al. (2013) who replaced the basic heat pump EnergyPlus object with a userdefined component based on quadratic equations gave a hint about this possible coupling.

This paper presents the methodology used for connecting an EnergyPlus building model to a real heat pump under a HIL configuration and the results of a two days' experiment performed in a semi-virtual testing laboratory (SEILAB), which features are described by Péan et al. (2019). During the experiment, real-time weather data and photovoltaic (PV) power production are provided to the simulation model. The entire communication chain is based on the use of the Functional Mock-up Interface (FMI) for Co-Simulation (Modelisar Consortium, 2010), on LabVIEW software (2018) and it could be implemented in other laboratories due to the standardization of the process.

\section{Methods}

Figure 1 represents the general scheme of the overall configuration. The real elements are the weather station, the PV installation and the water-to-water heat pump. Those components communicate with the building model implemented in EnergyPlus. The EnergyPlus model has been wrapped into a Functional Mock-up Unit (FMU) that communicates with the laboratory using LabVIEW. The latter provides a graphical interface of the laboratory to manage the real equipment. Coupling between EnergyPlus and LabVIEW requires Python's scripts as a bridge for communication. 
An experiment of two days has been performed to evaluate the behaviour of the data communication chain and to identify the opportunities and limitations of this solution. The experiment has been performed in a winter period, in particular on December $18^{\text {th }}-19^{\text {th }}$ and with a simulation time resolution of three minutes.
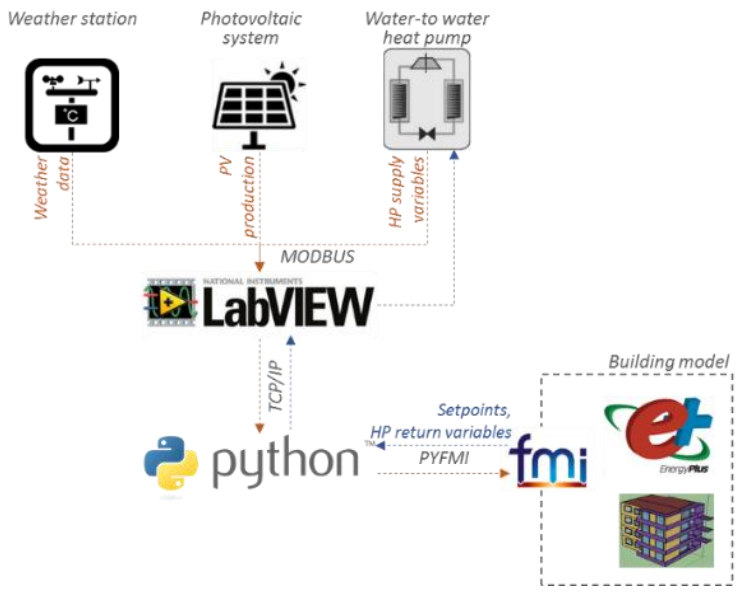

\section{Figure 1: General schematic of overall configuration}

A detailed description of each component, the communication between them and the results of the experiment is presented in the following sections.

\section{Building and systems description}

Building model developed in EnergyPlus environment represents a residential building located in Tarragona, Spain. It is representative of a Spanish multi-story building of the period from 1991 to 2007 and it follows the building code NRE-AT-87, which building typology is described by Tejero et al. (2018). It consists in four identical dwellings of two thermal zones, each one with a different occupancy level. In addition, occupancy, appliances and lighting consumptions follow stochastic profiles that differentiate each dwelling behavior, resulting in different energy demands. Regarding domestic hot water (DHW) profiles, they are based on the European standard (EN16147, 2011). It provides the energy consumption for different daily DHW usages and tapping profiles. In the present study, tapping cycle "medium" and equivalent DHW temperature of $60^{\circ} \mathrm{C}$ has been selected and adapted to the occupancy and simulation time step.

Heating is covered by means of a centralized water-towater geothermal heat pump system that provide hot water for the indoor fan coil units (two units per dwelling) and the DHW. The DHW system is composed by four storage systems, one for each household, that are modelled by a four node stratified tank. Heating loop circuit from the heat pump is connected to the bottom half part of the tanks and electrical heaters are placed on the top part acting as auxiliary systems. This configuration allows maintaining the recommended criterion regarding DHW temperature $\left(60^{\circ} \mathrm{C}\right)$. Space and DHW heating is controlled on three levels:

- Fan coils: thermostat temperatures of the thermal zones are compared with the actual temperatures sensed with the Energy Management System (EMS) sensors of EnergyPlus. A temperature hysteresis control with a deadband of $0.5^{\circ} \mathrm{C}$ is implemented with an internal program. The heat provided by the fan coils is directly managed with the EMS actuator Fan Coil Air Mass Flow Rate to mimic an on-off two-stages control. In addition, in order to communicate wih the heat pump, each time step the fan coils launch a binary signal, one if heating is needed in the zone or zero for the contrary case.

- DHW storage tanks: storage tanks present two thermostats, one in the top part (Node 1) and the other in the middle bottom part (Node 3). In this case, the EnergyPlus internal control is used. Node 1 temperature is controlled by an electrical heater and by an hysteresis control with a dead band of $2^{\circ} \mathrm{C}$. On the other hand, the bottom part is connected with the heat pump loop and is controlled by an hysteresis control with a dead band of $5^{\circ} \mathrm{C}$. As for the fan coils, storage tanks launch a binary signal to the heat pump based on the bottom part control.

- Heat pump: the heat pump presents two controls. An external one which come from the simulation and an internal one. The external one, called "heat pump status", is a global signal that is generated as summation of each individual binary signals that comes from the fan coils and from the storage tanks. If this global signal is greater than one, hot water flows through the main heating loop and the simulation sends an ON signal to the heat pump. On the other hand, the internal signal operates according to the condenser inlet temperature setpoint. In particular, when the condenser inlet temperature reaches the setpoint, the heat pump compressor is switched off.

A PV installation is included in the system. The real PV installation has a peak power of $3.75 \mathrm{kWp}$ but, for the purpose of the simulation, the power introduced to the building model has been scaled up to $10.8 \mathrm{kWp}$ to simulate a nearly Zero Energy Building (nZEB). Moreover, the building is equipped with a $10 \mathrm{kWh}$ community battery connected to a DirectCurrentWithInverterDCStorage load center. In order to enhance photovoltaic selfconsumption, the EnergyPlus storage operation scheme used is TrackFacilityElectricDemandStoreExcessOnSite.

Figure 2 shows the geometry of the building while Table 1 summarizes the main features of the building and its systems, highlighting the real and simulated elements.

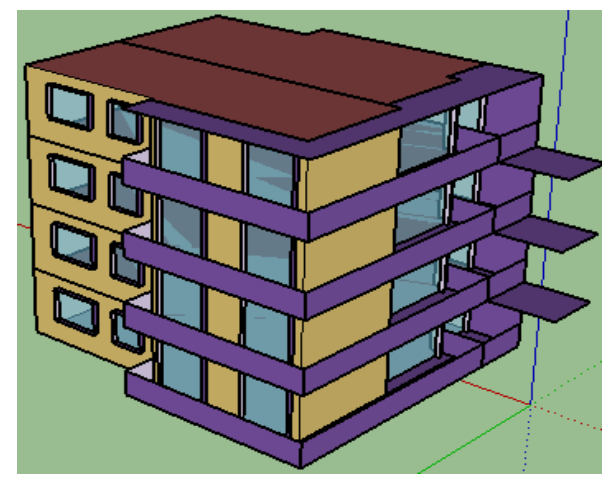

Figure 2: Geometry of the building model 
Table 1: Main building model features

\begin{tabular}{|c|c|c|}
\hline \multicolumn{2}{|c|}{ Building model features } & Real / Simulated \\
\hline Location & Tarragona, Spain & Real weather data \\
\hline $\begin{array}{l}\text { Year of } \\
\text { construction }\end{array}$ & 1990 & \multirow{4}{*}{ Simulated } \\
\hline $\begin{array}{l}\text { Constructive } \\
\text { features }\end{array}$ & $\begin{array}{l}\text { Following the } \\
\text { building code } \\
\text { NRE-AT-87 }\end{array}$ & \\
\hline $\begin{array}{l}\text { Number of } \\
\text { dwellings }\end{array}$ & 4 & \\
\hline $\begin{array}{l}\text { Surface } \\
\text { area/dwelling }\end{array}$ & $104.3 \mathrm{~m}^{2} /$ dwelling & \\
\hline Heating unit & $\begin{array}{c}\text { Centralized } \\
\text { geothermal heat } \\
\text { pump }\end{array}$ & $\begin{array}{l}\text { Real water-to- } \\
\text { water heat pump }\end{array}$ \\
\hline Heat sink & Ground Borehole & Simulated \\
\hline $\begin{array}{l}\text { Heat Emission } \\
\text { units }\end{array}$ & Fan-coil units & Simulated \\
\hline DHW system & $\begin{array}{l}\text { Dwelling tank + } \\
\text { electrical backup }\end{array}$ & Simulated \\
\hline Electric battery & $10 \mathrm{kWh}$ & Simulated \\
\hline Electric vehicle & $24 \mathrm{kWh}$ & Simulated \\
\hline PV system & $10.8 \mathrm{kWp}$ & $3.7 \mathrm{kWp}$ (real) \\
\hline
\end{tabular}

The main objective of the building model is to simulate the virtual components that are not present in the laboratory environment.

\section{Emulated systems in the Semi-virtual Energy Integration Laboratory (SEILAB)}

The objective of the communication interface between the model and the heat pump is to operate the laboratory equipment under a HIL configuration. Within this environment, the heat pump is operated under the dynamic thermal loads of the building that is implemented and simulated in the EnergyPlus model. The operation of the heat pump under dynamic loads allows characterizing its performance under quasi-realistic conditions that reproduce its behavior in real world scenarios.

The laboratory thermal test benches are used to emulate the ground thermal source and the building loads that are connected to the heat pump. This is done by means of a set of hydraulic loops equipped with the necessary controllers and sensors that allow for monitoring and controlling temperature and water flow in all the circuits (Table 2). Flow and temperature are controlled to reproduce the thermal energy profiles from the building model by means of circulation pumps equipped with frequency inverters, magnetive-inductive flow meters and high-speed modulating control valves provided with magnetic actuators. This control is done by means of valves that act by diverting water flow through by-pass lines, and heat exchangers that provide the heating and cooling requirements for each test bench to emulate heat sources and sinks. The electricity consumed by the heat pump is measured with a Siemens Sentron PAC3100 analyzer with an accuracy of $\pm 1 \%$ for the power measurement. The schematic of the laboratory experimental set up is shown in Figure 3.
Table 2: Precision specifications for laboratory temperature, water flow and pressure sensors

\begin{tabular}{|l|l|l|l|}
\hline \multicolumn{1}{|c|}{ Parameter } & Units & \multicolumn{1}{|c|}{ Device } & \multicolumn{1}{c|}{ Precision } \\
\hline Temperature & $\mathrm{K}$ & Pt 100 & $\pm 0.25 \mathrm{~K}$ \\
\hline Water flow & $\mathrm{m}^{3} / \mathrm{h}$ & Induction flowmeter & $\pm 0.2-0.5 \%$ \\
\hline Pressure & $\mathrm{bar}$ & Pressure transmitter & $\pm 1 \%$ \\
\hline
\end{tabular}

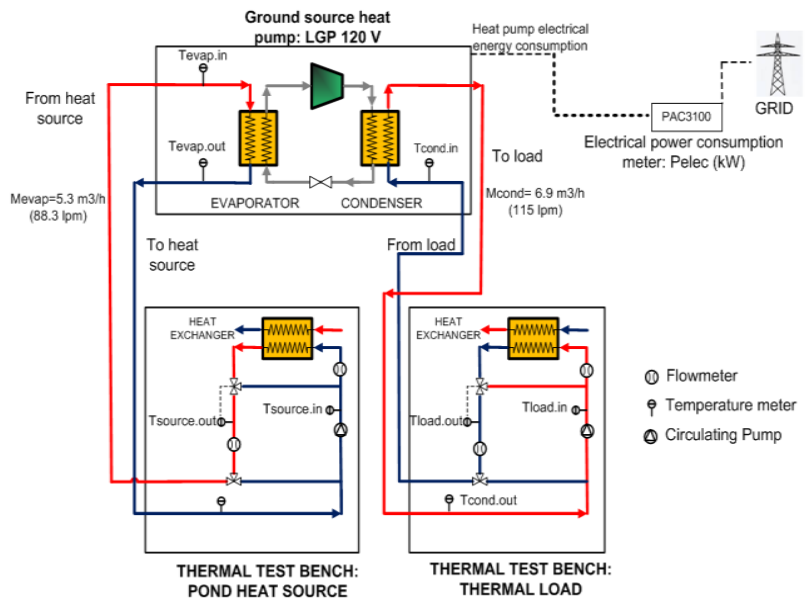

Figure 3: Scheme of laboratory set up for testing.

A fixed capacity water-to-water heat pump is used in the experiments performed in the SEILAB to provide heat to cover the heating and DHW needs of the virtual building. The DYNACIAT LGP $120 \mathrm{~V}$ heat pump installed in SEILAB is fully instrumented for the characterization of the thermal properties of the refrigerant and hydraulic circuits. The heat production and electrical energy consumption from the heat pump are measured and controlled by means of high precision measurement and control elements installed in the laboratory test benches. The technical characteristics of the heat pump used for the project are described in Table 3.

Table 3: Technical characteristics of the heat pump under study. *Performance data according to EN14511

\begin{tabular}{|c|c|}
\hline Parameter & Value \\
\hline Net heating capacity*: & $40.5 \mathrm{~kW}$ \\
\hline Net power input*: & $10.1 \mathrm{~kW}$ \\
\hline Net COP* & $4.02 \mathrm{~kW}$ \\
\hline Compressor type & Hermetic scroll $(2900 \mathrm{rpm})$ \\
\hline Refrigerant type: & $\mathrm{R} 410 \mathrm{~A}$ \\
\hline Electrical supply type & 3-phase AC $50 \mathrm{~Hz}-400 \mathrm{~V}$ \\
\hline Evaporator type: & Brazed-plate heat exchanger \\
\hline Evaporator flow range: & $3.5-11.2 \mathrm{~m}^{3} / \mathrm{h}$ \\
\hline $\begin{array}{c}\text { Evaporator water outlet } \\
\text { temperature range: }\end{array}$ & $-10^{\circ} \mathrm{C} /+18^{\circ} \mathrm{C}$ \\
\hline Condenser type: & Brazed-plate heat exchanger \\
\hline Condenser flow range: & $3.1-8.5 \mathrm{~m} 3 / \mathrm{h}$ \\
\hline $\begin{array}{c}\text { Condenser water outlet } \\
\text { temperature range: }\end{array}$ & $30^{\circ} \mathrm{C} / 55^{\circ} \mathrm{C}$ \\
\hline Dimensions & $1.2 \mathrm{mx} 0.798 \mathrm{mx} 0.883 \mathrm{~m}$ \\
\hline Weight & $240 \mathrm{~kg}$ \\
\hline
\end{tabular}

On the roof platform of the SEILAB building, a meteorological station is placed to obtain measurements of outdoor ambient. The meteorological station measures dry bulb temperature, relative humidity, wind direction, wind speed, global solar radiation and diffuse solar 
radiation. The station communicates in real-time with the laboratory via Modbus protocol and has a data logger for a backup of data. More information about the measurement instruments can be found in Table 4 .

Table 4: Precision specifications for weather station equipment

\begin{tabular}{|c|c|c|c|c|}
\hline Measurement & Brand & Units & Range & Accuracy \\
\hline Wind speed & Gill & $\mathrm{m} / \mathrm{s}$ & $\begin{array}{l}0-60 \\
\mathrm{~m} / \mathrm{s}\end{array}$ & $\pm 2 \%$ at $12 \mathrm{~m} / \mathrm{s}$ \\
\hline Wind direction & Gill & o & $0-359^{\circ}$ & $\pm 3^{\circ}$ at $20 \mathrm{~m} / \mathrm{s}$ \\
\hline $\begin{array}{l}\text { Dry bulb } \\
\text { temperature }\end{array}$ & $\begin{array}{l}\text { Campbell } \\
\text { Scientific }\end{array}$ & ${ }^{\circ} \mathrm{C}$ & $\begin{array}{l}- \\
39.2^{\circ} \mathrm{C} \\
\text { to } \\
+60^{\circ} \mathrm{C}\end{array}$ & $\pm 0.2 \%$ at $20^{\circ} \mathrm{C}$ \\
\hline $\begin{array}{l}\text { Relative } \\
\text { humidity }\end{array}$ & $\begin{array}{l}\text { Campbell } \\
\text { Scientific }\end{array}$ & $\%$ & $\begin{array}{l}0.8 \text { to } \\
100 \% \\
\text { RH }\end{array}$ & $\begin{array}{lll} \pm 1 \% & \text { RH } & \text { at } \\
20^{\circ} \mathrm{C} & \end{array}$ \\
\hline $\begin{array}{l}\text { Global } \\
\text { Radiation }\end{array}$ & Kipp\&Zonen & $\mathrm{W} / \mathrm{m} 2$ & $\begin{array}{l}0-2000 \\
\mathrm{~W} / \mathrm{m} 2\end{array}$ & $\begin{array}{l}<20 \mathrm{~W} / \mathrm{m} 2 \text { at } \\
80^{\circ} \text { with } 1000 \\
\mathrm{~W} / \mathrm{m} 2 \text { irradiance }\end{array}$ \\
\hline $\begin{array}{l}\text { Diffuse } \\
\text { radiation }\end{array}$ & $\begin{array}{l}\text { Delta-T } \\
\text { Devices }\end{array}$ & $\mathrm{W} / \mathrm{m} 2$ & $\begin{array}{l}0-2000 \\
\mathrm{~W} / \mathrm{m} 2\end{array}$ & $\begin{array}{l} \pm 2 \% \\
\text { incoming } \\
\text { radiation }\end{array}$ \\
\hline
\end{tabular}

The PV system is placed on the roof of the building of the laboratory (Figure 4). The PV system consists of 14 polycrystalline solar panels, brand model REC FV $245 \mathrm{Wp}$ as it is shown in Figure 4. It has a total area of $23.1 \mathrm{~m}^{2}$ and a total peak power installed of $3.75 \mathrm{kWp}$. The PV system is connected to an inverter of the brand SMA model SB 3600 TL with the technical specifications described in Table 5.

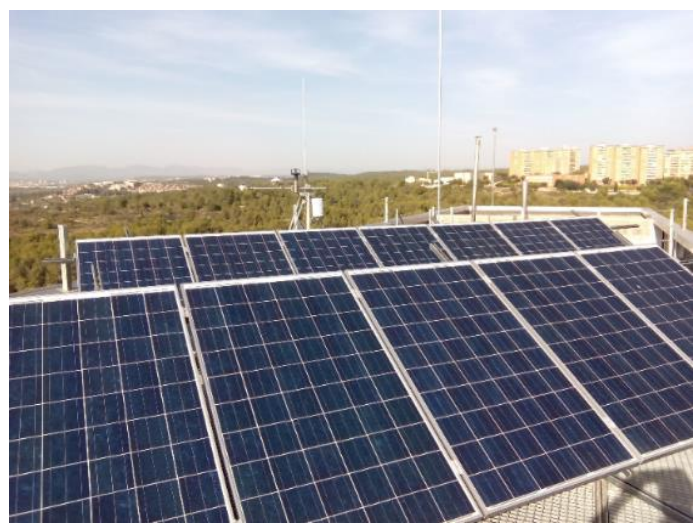

Figure 4: PV system on the roof of the SEILAB building

The tests performed in SEILAB are supervised and controlled by a data acquisition and control system created with the software LabVIEW. Communications between the supervisory system and the laboratory components are based on Modbus RTU, through RS485 connections and on Modbus TCP/IP. The communication with the heat pump equipment is done via Modbus RTU, while the communication with the meteorological station and the PV installation is done via Modbus TCP

Table 5: PV system operation values

\begin{tabular}{|c|c|}
\hline \multicolumn{2}{|c|}{ Input (DC) } \\
\hline Max. DC power (at $\cos \varphi=1$ ) & $3880 \mathrm{~W}$ \\
\hline Max. input voltage & $750 \mathrm{~V}$ \\
\hline \multicolumn{2}{|c|}{ Output (AC) } \\
MPP voltage range / rated input voltage & $175 \mathrm{~V}$ to $500 \mathrm{~V} /$ \\
\hline \multicolumn{2}{|c|}{$400 \mathrm{~V}$} \\
\hline Rated power (at 230 V, 50 Hz & $3680 \mathrm{~W}$ \\
\hline Max. AC apparent power & $3680 \mathrm{VA}$ \\
\hline
\end{tabular}

\begin{tabular}{|c|c|}
\hline Nominal AC voltage / range & $\begin{array}{c}220 \mathrm{~V}, 230 \mathrm{~V}, 240 \\
\mathrm{~V} / 180 \mathrm{~V} \text { to } 280 \mathrm{~V}\end{array}$ \\
\hline AC power frequency / range & $\begin{array}{c}50 \mathrm{~Hz}, 60 \mathrm{~Hz} /-5 \\
\mathrm{~Hz} \mathrm{to}+5 \mathrm{~Hz}\end{array}$ \\
\hline Rated power frequency / rated grid voltage & $50 \mathrm{~Hz} / 230 \mathrm{~V}$ \\
\hline Max. output current & $16 \mathrm{~A}$ \\
\hline Max. efficiency / European Efficiency & $97 \% / 96,4 \%$ \\
\hline
\end{tabular}

EnergyPlus integration with the Functional Mock-up Interface

To perform the real-time data exchange with the laboratory, the EnergyPlus model has been wrapped into an FMU using the EnergyPlusToFMU software package (Release 2.0.3, available on http://simulationresearch.lbl.gov/). As explained by Pang et al. (2016), this software reads the EnergyPlus input file (IDF), the Input Dictionary file (IDD), the weather file (EPW) and generates an FMU that is ready for cosimulation. To interface EnergyPlus with the Functional Mock-up Interface (FMI), the IDF file has to be configured using a specific model called ExternalInterface:FunctionalMockupUnitExport. This model allows adding new objects that work in the same way of the basic EnergyPlus schedules (To:Schedule), EMS actuators (To:Actuator) and EMS variables (To: Variable) objects, as explained by Pang et al. (2012) and in the ExternalInterfacesApplicationGuide of EnergyPlus.

In this study, the objects To:Variable and To:Actuator are used to provide external inputs to the model such as realtime weather data, PV production, temperatures setpoints and heat pump variables. On the other hand, the object From:Variable is used to export EnergyPlus time step calculation results. Figure 5 represents the architecture of the integration between the software acting in a master and slave configuration.

Meanwhile the procedure to exchange variables such as setpoints schedules or weather data is quite straightforward and already discussed by Pang (2016), the HIL co-simulation with a real heating unit and real renewable energy system present some points that is worth to discuss. EnergyPlus HVAC systems need to be a closed loop. This means that it is not possible to remove the heat pump object from the model and simply exchange data with the real heat pump temperature and power sensors, as it could be implemented in other dynamic simulation software as TRNSYS, as explained by Fuentes et al. (2018). To overtake the obstacle, the heat pump object has been replaced with a UserDefined component. This is a custom component available in EnergyPlus that can be programmed using the EMS. By the use of EMS variables, actuators and the external interface, it is possible to exchange the main variables of the heat pump. The EnergyPlus model provides temperatures and mass flows that have to be transferred to the heat pump evaporator and condenser inlets from the borehole and the building heating distribution system, respectively. Then, after the time step in which the real equipment is under operation, outlet temperatures and mass flows are transferred to the simulation model. These are the mass 
flows and inlet temperatures to the borehole and the heating distribution system coming from the outlet of the evaporator and condenser, respectively.

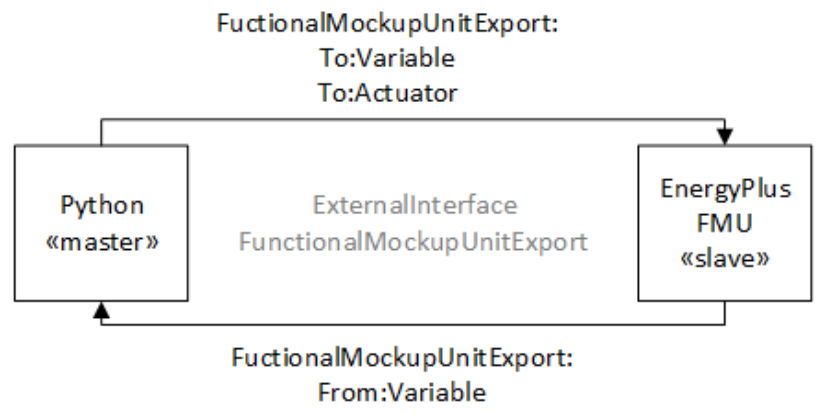

Figure 5: Master and Slave integration to communicate EnergyPlus with the laboratory equipment

Figure 6 represents a schematic of the process, showing how the temperatures measured at the outlet of the real heat pump condenser and evaporator overwrite UserDefined's internal variables and pass the values to system nodes. On the other hand, system node temperatures resulting from the simulation loop are sent to the real equipment.

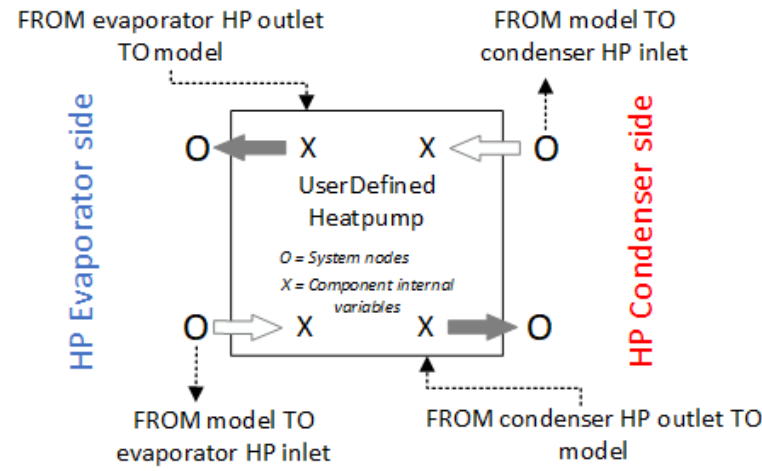

Figure 6: UserDefined Heat Pump in EnergyPlus

Regarding the PV production, EnergyPlus does not allow using the actuator called On-Site Generator Control Requested Power directly with Generator:Photovoltaic object to overwrite the renewable energy production. To do that, PV object has been successfully replaced with Generator:MicroTurbine object, which allows overwriting the production of the model with an external value provided through the FMU. In this way, the externally measured renewable energy is imposed as generator requested energy and it can be injected in the electrical load distribution centre of EnergyPlus. Table 6 shows the most relevant variables exchanged between the model and the laboratory. Those one that are "from" EnergyPlus are outputs of the FMU meanwhile those one that are "to" EnergyPlus are inputs of the FMU. On the other hand, setpoints, which are set through the lab, can be either fixed, variables through schedules or variables along time depending on the result of basic/advanced control actions.
Table 6: Exchanged variables between SEILAB and

\begin{tabular}{|c|c|c|}
\hline \multicolumn{3}{c}{ EnergyPlus } \\
\hline $\begin{array}{c}\text { Inlet condenser } \\
\text { temperature }\end{array}$ & EnergyPlus & SEILAB \\
\hline $\begin{array}{c}\text { Outlet condenser } \\
\text { temperature }\end{array}$ & SEILAB & EnergyPlus \\
\hline $\begin{array}{c}\text { Inlet evaporator } \\
\text { temperature }\end{array}$ & EnergyPlus & SEILAB \\
\hline $\begin{array}{c}\text { Outlet evaporator } \\
\text { temperature }\end{array}$ & SEILAB & EnergyPlus \\
\hline Heat pump status & EnergyPlus & SEILAB \\
\hline $\begin{array}{c}\text { Outlet condenser } \\
\text { temperature setpoint }\end{array}$ & EnergyPlus & SEILAB \\
\hline $\begin{array}{c}\text { Bottom water tank } \\
\text { setpoints }\end{array}$ & External & EnergyPlus \\
\hline $\begin{array}{c}\text { Air zone temperature } \\
\text { setpoints }\end{array}$ & External & EnergyPlus \\
\hline Weather data & SEILAB & EnergyPlus \\
\hline PV power & SEILAB & EnergyPlus \\
\hline
\end{tabular}

\section{FMU connection with Python and LabVIEW}

In this project there are two data flows, one that communicates the data from the laboratory's sensors and real equipment to the FMU model, and the other that communicates the data from the model to LabVIEW. Coupling project's FMU with LabVIEW directly presented some difficulties. There are certain libraries for using FMU and LabVIEW, but, depending on the licenses, the type of FMU has to be model exchange. The solution adopted here uses Python as the FMU master. Using the Python platform Anaconda and the library FMUpy, a script for command the FMU is created. The values of the start_time, step_time and stop_time of the script and the simulation model must be the same. For the communication between Python and LabVIEW, a TCP/IP communication port is generated. This port is used for the data exchange between the model and the laboratory.

The procedure is described within the following points and represented in Figure 7 :

- First: Python, actuating as a server, creates the $\mathrm{TCP} / \mathrm{IP}$ connection in a specific port of the computer and initializes the simulation model. The model starts with the warming up. Once the model has finished the warming up, there is a delay of time of the time step of the simulation (three minutes).

- Second: After these three minutes, LabVIEW sends an array of data (measured values) to Python through the TCP/IP line.

- Third: The data is received by the Python script, each value is separated and assigned to the corresponding variable. Then a time step is performed in the simulation model.

- Forth: This time step generates the outputs from the simulation. Those outputs are aggregated into an array and sent by TCP/IP port.

- Fifth: LabVIEW gets the outputs from the simulation and assigns these values to the corresponding variables. 
- Sixth: After a delay of the time step, it returns to the second step of the process until it reaches the stop time defined in the FMU.

The delay of the time step is needed to synchronise the simulation with the real-time. All the data exchanged are stored in a local database in LabVIEW.

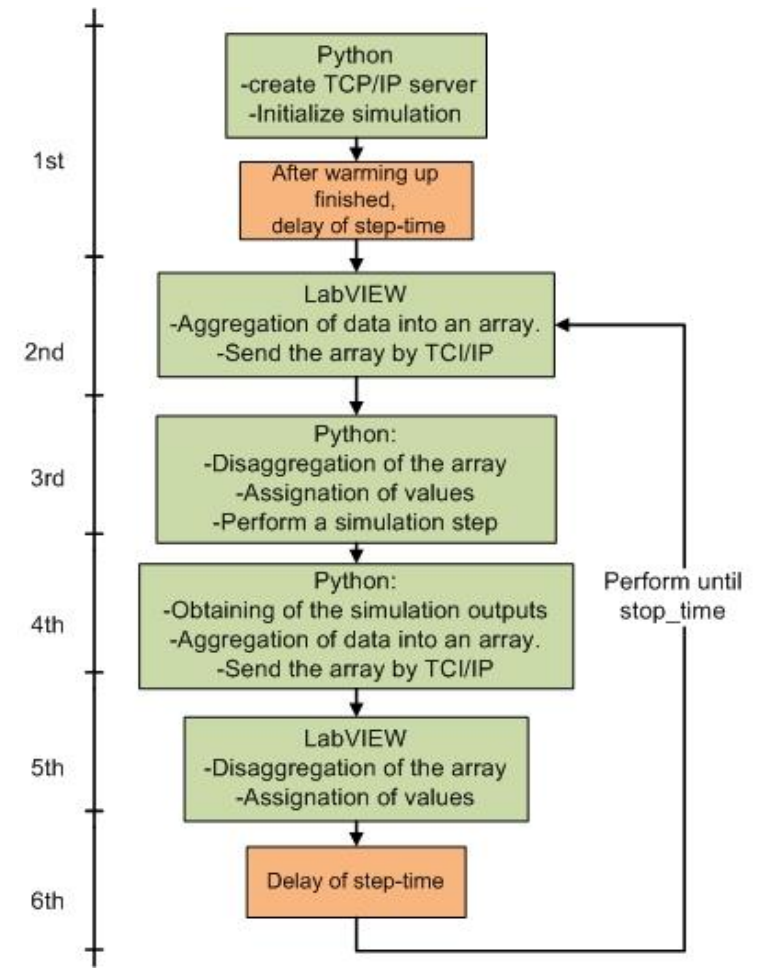

Figure 7: Communication scheme between FMU, Python and LabVIEW

\section{Results}

In this section, the results of the experiment are presented. The test was performed during two days in December, starting at 00:00h of $18^{\text {th }}$ and finishing at 00:00h of the $20^{\text {th }}$. The analysed variables aim to show that the exchange of data between the building model and the real equipment is working properly, and the performance of the building simulation is the expected one.

After the experiment execution, a problem has been identified with the initialization of the setpoint temperature of the zones, which is an input of the FMU. Despite there is a field in the FMU code to define the initial value of the input variables (ExternalInterface:FunctionalMockupUnitExport:To:Act uator, Initial Value), EnergyPlus seems not using this value in the warm up process of the simulation. As a result of this problem, the initial value of the zones is $5-7^{\circ} \mathrm{C}$ (instead of $18^{\circ} \mathrm{C}$ ), and the simulation needs around $24 \mathrm{~h}$ to stabilize the temperature to the setpoint of the zone $\left(18^{\circ} \mathrm{C}\right.$ at night and $21^{\circ} \mathrm{C}$ during the day). However, after this first period of simulation, that could be accelerated through the python script, zones temperatures perfectly follow the setpoint and the hysteresis control programmed with the EMS.

The evaluation of the experiment is done for a period when the simulation is already stabilized, and the shown results correspond to the late first and second day experiment (December $18^{\text {th }}$ and $19^{\text {th }}$ ). However, graphs are time-scaled according to the level of accuracy that authors consider the appropriate one. In particular, heat pump operation is shown for a four-hour period meanwhile zone temperatures, fan coils power and DHW demand for one day and the PV behaviour for the first hours of the morning.

The evolution of the heat pump condenser variables is shown in Figure 8. The heat pump receives the external signal for operation (heat pump status), while its internal control decides whether to start or stop the heat pump compressor depending on the inlet temperature of the condenser. The internal setpoint of the heat pump on the condenser side is $50^{\circ} \mathrm{C}$, which value comes from the FMU. Tcond.in setpoint is the temperature that the laboratory has to emulate, representing the loss in temperature of the fluid due to the heating demand of the building. This temperature setpoint is achieved with the flow of the fluid through a heat exchanger (test benches). This operation is controlled by a PID loop control that commands a high precision control valve. On the other hand, the inlet temperature from the fan coils to the condenser is controlled by the regulation system in the laboratory with little deviation with respect to the setpoint. The temperature lift between the inlet and outlet temperatures of the condenser is approximately $4^{\circ} \mathrm{C}$.

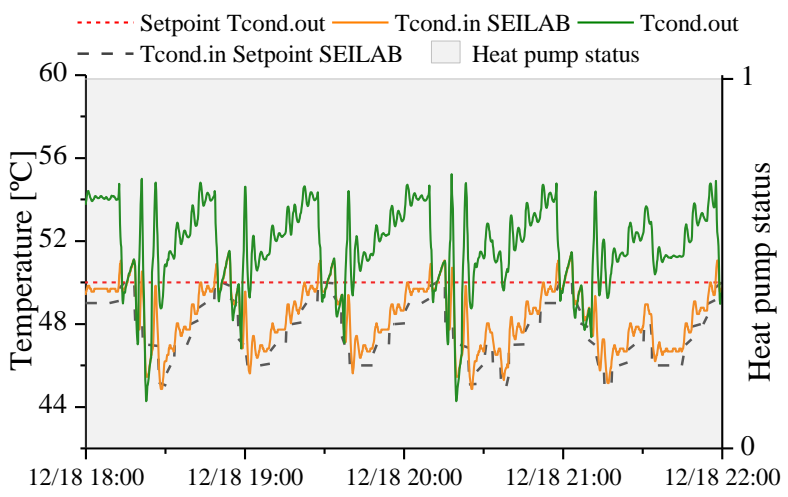

Figure 8: Evolution of temperatures at the condenser of the heat pump

Similarly, the evolution of the variables in the evaporator are shown in Figure 9, in particular the inlet and outlet temperatures. The control of the inlet temperature from the ground source is good with values between 10 and 14 ${ }^{\circ} \mathrm{C}$.

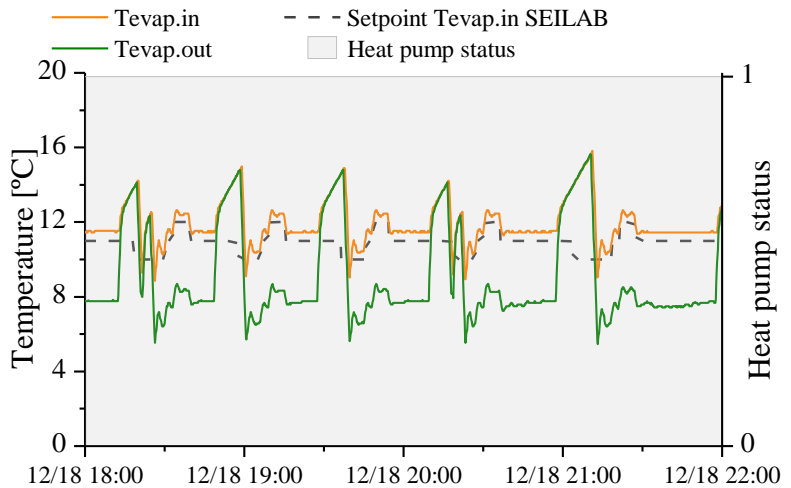


Figure 9: Evolution of temperatures at the evaporator of

$$
\begin{aligned}
& \text { the heat pump } \\
\text { - Qcond } & \text { Electric power }
\end{aligned}
$$

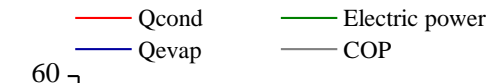

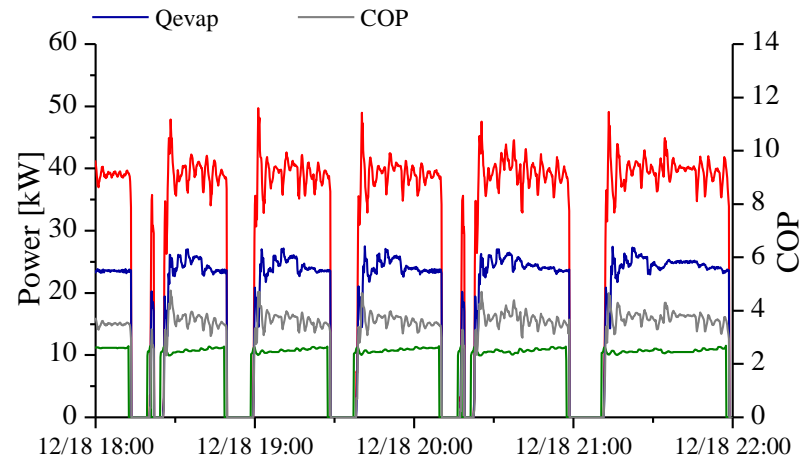

Figure 10: Evolution of COP, thermal and electrical power of heat pump

The trends of the thermal and electrical power variables are shown in Figure 10. The fixed-capacity heat pump operates in an on-off cycling trend in stages from 20 to 45 minutes approximately. The electrical power consumption is between 10 and $12 \mathrm{~kW}$, while the COP value oscillates near four.

Figure 11 shows the thermal behaviour of the first floor dwelling for one-day experiment. Both day and night zones temperatures, the common setpoint and the outdoor air temperature are represented in the left-axis. These data are complemented with the thermal load of the fan coils, represented in the right-axis. The figure shows how the air temperature of the zones are able to follow the setpoint, which value is an input of the FMU. Additionally, it is possible to relate the behaviour of the air zone temperatures to the heat provided by the fan coils. It can be observed three different levels of thermal power, according to the temperature of the two zones: the fan coils are switched-off, there is one fan coil working (around $3 \mathrm{~kW}$ ), and there are two fan coils working (around $6 \mathrm{~kW}$ ). Moreover, the slight delay with which temperatures follows the setpoint during setpoint variation highlights zones capacitance.

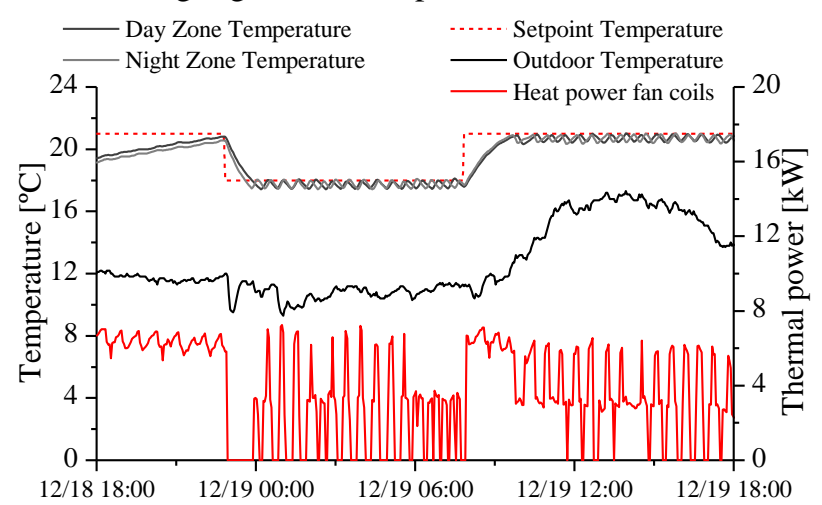

Figure 11: Temperatures and thermal power of the thermal zones of the first floor dwelling

Figure 12 shows the thermal behaviour of the hot water storage tank of the first floor dwelling and its associated DHW draw profile. Average tank temperature, node 1 (top tank) temperature setpoint and node 3 temperature setpoint are represented in the left axis. In the right axis is reported the accumulated energy for the DHW demand, the thermal energy that comes from the heat pump, the one from the electric resistance and the DHW extraction in liters per minute. DHW demand is evaluated taking into account the water mass flow drawn for the consumption and the temperature difference between the main water temperature $\left(15^{\circ} \mathrm{C}\right)$ and the consumption one $\left(60^{\circ} \mathrm{C}\right)$. Regarding the control, temperature setpoint of node 3 controls the heat pump heat supply meanwhile temperature setpoint of node 1 controls the auxiliary resistance heat supply. When there is a DHW drawn due to a consumption, cold water enters to the tank and tank temperature falls down. This is the main reason why the two heating elements turn on. It could also happen when storage tank temperature falls down due to thermal losses. It can be observed a peak DHW consumption around $21: 00 \mathrm{~h}$ of the $18^{\text {th }}$ that is associated to a hot shower.

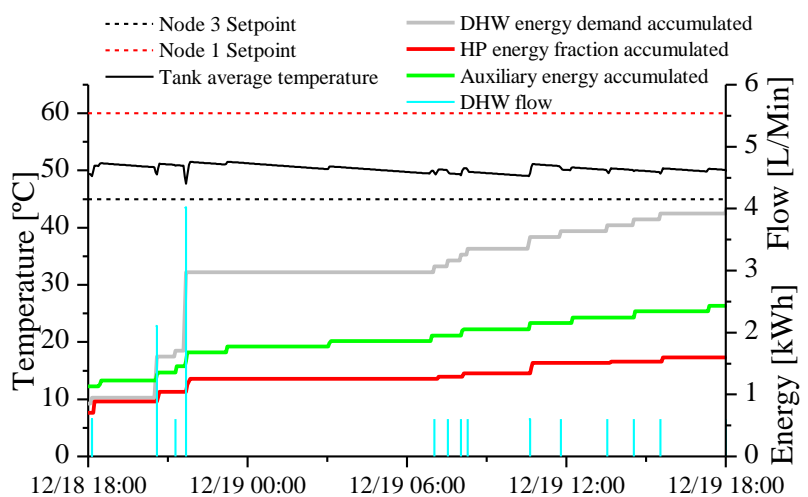

Figure 12: Temperatures and thermal power of the thermal zones of the first floor dwelling

Regarding the real PV system integration, Figure 13 shows the global irradiation measured by the on-site weather station (left axis), the power measured by the laboratory sensors and the output reported by EnergyPlus (right axis). This latter is scaled up as commented before. PV production clearly follows global irradiation as it could be expected. It could be noticed that measured power and EnergyPlus output do not perfectly match. This is due to the fact that EnergyPlus uses a higher time step than the laboratory measurements, since these latter have a precision of ten seconds while the FMU communication happens each three minutes.

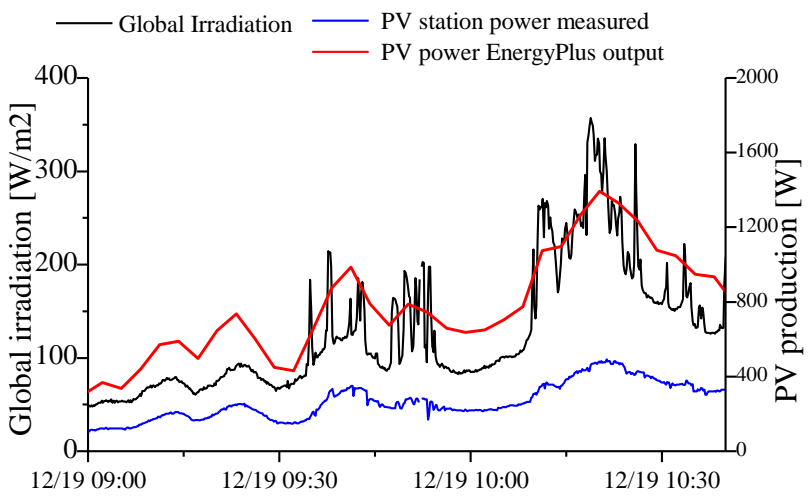

Figure 13: Evolution of the global irradiation and PV powers 


\section{Conclusion}

Few studies exist that tackle the communications of real equipment with simulation models in HIL configurations. This study deals with the operation of a heat pump, a weather station and a PV system in a HIL configuration and the connections required to create the experimental setting. Connections are done between an EnergyPlus model and the laboratory equipment by means of FMI communication. The methods used for establishing the data transfer for co-simulation are described and experiments are conducted to illustrate the operation of the overall system.

As a conclusion, the study shows that the temperature control implemented and the HIL integration work in an effective way ensuring comfortably temperatures in the thermal zones, the recommended temperature for DHW and a proper heat pump operation. Communication protocol between the laboratory, the PV installation and the weather station have been validated, as well as the communication protocol between the real heat pump and the building model. The FMI communication is able to transfer correctly the PV power production, the weather data, the heat pump main variables and all the setpoints introduced. Further studies will involve the implementation of detailed control strategies, as model predictive control (MPC), instead of scheduled setpoints, in order to enhance energy flexibility in buildings.

\section{Acknowledgement}

This project has received funding from the European Union's Horizon 2020 research and innovation programme under the H2020-LCE-01-2016-2017 grant agreement No 731211 (SABINA).

We would like to thank to University of Navarra for the support in the modelization of the building model in EnergyPlus.

\section{References}

Consortium, M. (2010). Functional Mock-up Interface for Co-Simulation, available from: https://fmistandard.org/

E-Baz W., Mayerhofer L, Tzscheutschles P., Wagner U. (2018). Hardware in the Loop Real-Time Simulation for Heating Systems: Model Validation and Dynamics Analysis. Energies 11, 3159.

EnergyPlus (2016). External Interface(s) Application Guide, Guide for using EnergyPlus with External Interface.

European Standard, EN 16147, January 2011. Heat pumps with electrically driven compressors-Testing and requirement for marking of domestic hot water units, January 2011.

Fuentes E., Impala V., Bellanco I., Salom J., Sayans A., Balsells M., Sanjoaquin S. and Mogro O. (2018). Experimental investigation on the dynamic performance of a solar thermally-driven adsorption chiller integrated with a gas boiler. Proceedings from
EuroSun. Rapperswill (Switzerland), 10-13 September 2018.

Jones T. A., Finn D. P. (2013). Ground source heat pump modelling with thermal storage- simulation and integration issues in Energy Plus. Proceedings of BS2013: 13th Conference of International Building Performance Simulation Association. Chambéry (France), 26-28 August 2013.

National Instrument, LabVIEW 2018. Information available on: http://www.ni.com/eses/shop/labview/labview-details.html

Pang X., Nouidui T. S., Wetter M., Fuller D., Liao A. et Haves P. (2016). Building energy simulation in real time through an open standard interface. Energy and Buildings 117, 282-289.

Pang X., Wetter M., Bhattacharya P. et Haves P. (2012). A framework for simulation-based real-time whole building performance assessment. Building and Environment 54, 100-108.

Péan T., Salom J. (2019). Laboratory facilities used to test energy flexibility in buildings. A technical report from IEA EBC Annex 67 Energy Flexible Buildings, Second Edition.

Tejero A., Ortiz J., Salom J. (2018). Evaluation of occupancy impact in a residential multifamily nZEB through a high resolution stochastic model. Proceedings of BSO 2018: 4th Building Simulation and Optimization Conference. Cambridge (UK), 1112 September 2018

Thierry S. Nouidui, D.M.L., Wetter M. (2018), EnergyPlusToFMU.

Waddicor D. A., Fuentes E., Azar M., Salom J. (2016). Partial load efficiency degradation of a water-to-water heat pump under fixed set-point control. Applied Thermal Engineering 106, 275-285. 